EPJ manuscript No.

(will be inserted by the editor)

\title{
Weak form factors for semileptonic octet baryon decays in the chiral quark model
}

\author{
T. Ohlsson ${ }^{\mathrm{a}}$, H. Snellman ${ }^{\mathrm{b}}$ \\ Theoretical Physics, Department of Physics, Royal Institute of Technology, SE-100 44 Stockholm, Sweden
}

Received: 9 March 1998

\begin{abstract}
We study the weak vector and axial-vector form factors of first- and second-class currents for the semileptonic octet baryon decays in the spirit of the chiral quark model. Our results for the weak magnetism form factors are consistent with the conserved vector current (CVC) results. The induced pseudotensor form factors, which are highly model dependent, are small. The overall performance of the chiral quark model is quite good and in general agreement with existing experimental data.
\end{abstract}

\section{Introduction}

The weak axial-vector form factors of the baryons have been, and still are, an important set of parameters for the investigation of their quark spin structure. In particular the so called "nucleon spin crisis" [1, 2], as an indication of the subtle dynamics of the quark spin polarization of the nucleons, relate the measurement of the deep inelastic scattering (DIS) parameters to the spin polarizations of the quarks via the baryonic axial-vector form factors.

The analysis from experiments of these form factors are normally performed assuming that the second-class form factors are negligible. However, for strangenesschanging currents between unequal mass states, the SU(3) symmetry breaking may induce non-negligible secondclass currents. At least one experiment have reported substantial such currents [3].

Since the axial-vector form factors are used for extracting the quark spin content of baryons, their exact values are of importance. Also when one wants to compare the axial-vector form factors with the Cabibbo theory, or with model calculations, it is important not to have a mixture of first- and second-class form factors to deal with.

Pending further experiments, it is of interest to estimate these form factors theoretically. This has been done earlier in a relativistic quark model [4], in the MIT bag model [5, 6, 7, 8], and in the MIT bag model with one-gluon QCD corrections [9]. Recently, there has also been a calculation within the chiral quark-soliton model [10]. Unfortunately, previous results do not mutually agree on the size of the $\Delta S=1$ second-class form factors, and in fact not even on the sign of them. This probably indicates that they are model dependent.

\footnotetext{
a e-mail: tommy@theophys.kth.se

b e-mail: snelletheophys.kth.se

Correspondence to: T. Ohlsson
}

In this paper, we estimate all six vector and axialvector form factors $f_{i}$ and $g_{i}$, where $i=1,2,3$, (defined in Sect. 2 below) in the spirit of the chiral quark model $(\chi \mathrm{QM})[11,12,13$ to linear order in the $\mathrm{SU}(3)$ symmetry breaking masses. This is of interest since the $\chi \mathrm{QM}$ gives a fair description of the magnetic moments of the baryons, and can be used to calculate the axial-vector form factors of the baryons in a way that substantially deviates from the non-relativistic quark model (NQM) due to the depolarization of the quark spins in the $\chi \mathrm{QM}$ by the Goldstone bosons (GBs). Our estimates are made in the same approximation as those of the magnetic moments of the baryons, treated earlier in the literature [14, 15, 16].

Besides the axial-vector form factor $g_{A} \equiv g_{1} / f_{1}$ our study will focus on the ratio $f_{2} / f_{1}$ for the vector current and the corresponding ratio $g_{2} / g_{1}$ for the axial-vector current. In the $\chi \mathrm{QM}$, the ratio $g_{2} / g_{1}$ is dependent only on the mass parameters. As shown by Donoghue and Holstein [5], the form factor $g_{2}$ is essentially the axial dipole moment, which is inversely proportional to the quark masses.

In our study, we find that the second-class form factors are small and highly model dependent. It seems therefore even more important to measure them, in order to find out more about the detailed dynamics of the baryons. It makes it also possible to directly compare the measured axial-vector form factors with the theoretical ones.

The outline of the paper is as follows. In Sect. 2 we describe the formalism and present the formulas for the first- and second-class form factors. In Sect. 3 we make estimates of the form factors in the $\chi \mathrm{QM}$. We end this section with a discussion about our results compared to other models and experiments. Finally, in Sect. 4 we present a summary and our conclusions. 


\section{Formalism}

\subsection{The weak form factors}

The transition matrix element $\mathcal{M}_{B \rightarrow B^{\prime} l^{-} \bar{\nu}_{l}}$ for the decay $B \rightarrow B^{\prime}+l^{-}+\bar{\nu}_{l}\left(q \rightarrow q^{\prime}+l^{-}+\bar{\nu}_{l}\right)$, is given by

$$
\mathcal{M}_{B \rightarrow B^{\prime} l^{-} \bar{\nu}_{l}}=\frac{G}{\sqrt{2}} V_{q q^{\prime}}\left\langle B^{\prime}\left(p^{\prime}\right)\left|J_{\text {weak }}^{\mu}\right| B(p)\right\rangle L_{\mu},
$$

where $G$ is the Fermi coupling constant, $V_{q q^{\prime}}$ is the $q q^{\prime}$ element of the Cabibbo-Kobayashi-Maskawa mixing matrix, and $L_{\mu}$ is the leptonic current.

The hadronic weak current is

$$
J_{\text {weak }}^{\mu}=J_{V}^{\mu}-J_{A}^{\mu}
$$

where $J_{V}^{\mu}$ is the vector current and $J_{A}^{\mu}$ is the axial-vector current. The matrix element of the vector current in momentum space of the transition $B \rightarrow B^{\prime}+l^{-}+\bar{\nu}_{l}$ is given by

$$
\begin{aligned}
\left\langle B^{\prime}\left(p^{\prime}\right)\right| J_{V}^{\mu} & |B(p)\rangle=\bar{u}^{\prime}\left(p^{\prime}\right)\left(f_{1}\left(q^{2}\right) \gamma^{\mu}\right. \\
& \left.-i \frac{f_{2}\left(q^{2}\right)}{M_{B}+M_{B^{\prime}}} \sigma^{\mu \nu} q_{\nu}+\frac{f_{3}\left(q^{2}\right)}{M_{B}+M_{B^{\prime}}} q^{\mu}\right) u(p)
\end{aligned}
$$

and the matrix element of the axial-vector current by

$$
\begin{aligned}
& \left\langle B^{\prime}\left(p^{\prime}\right)\left|J_{A}^{\mu}\right| B(p)\right\rangle=\bar{u}^{\prime}\left(p^{\prime}\right)\left(g_{1}\left(q^{2}\right) \gamma^{\mu} \gamma^{5}\right. \\
& \left.-i \frac{g_{2}\left(q^{2}\right)}{M_{B}+M_{B^{\prime}}} \sigma^{\mu \nu} q_{\nu} \gamma^{5}+\frac{g_{3}\left(q^{2}\right)}{M_{B}+M_{B^{\prime}}} q^{\mu} \gamma^{5}\right) u(p),
\end{aligned}
$$

where $M_{B}\left(M_{B^{\prime}}\right), p\left(p^{\prime}\right), u(p)\left(u^{\prime}\left(p^{\prime}\right)\right)$, and $|B(p)\rangle$ $\left(\left|B^{\prime}\left(p^{\prime}\right)\right\rangle\right)$ are the mass, momentum, Dirac spinor, and external baryon state of the initial (final) baryon $B\left(B^{\prime}\right)$, respectively, and $q=p-p^{\prime}$ is the momentum transfer [17]. The functions $f_{i}\left(q^{2}\right), i=1,2,3$, are the vector current form factors and the functions $g_{i}\left(q^{2}\right), i=1,2,3$, are the axial-vector current form factors. The form factors are Lorentz scalars and they contain all the information about the hadron dynamics. $f_{1}$ is the vector form factor, $f_{2}$ is the induced tensor form factor (or weak magnetism form factor or anomalous magnetic moment form factor), $f_{3}$ is the induced scalar form factor, $g_{1}$ is the axial-vector form factor, $g_{2}$ is the induced pseudotensor form factor (or weak electric form factor), and $g_{3}$ is the induced pseudoscalar form factor.

Under $G$-parity, the form factor $f_{2}$ transforms with the same sign as the form factor $f_{1}$, whereas the form factor $f_{3}$ has the opposite sign, and the form factor $g_{3}$ transforms with the same sign as the form factor $g_{1}$, whereas the form factor $g_{2}$ has the opposite sign. The currents with form factors $f_{3}$ and $g_{2}$ are therefore called second-class currents, and the others are first-class currents [18].

If we use the Gordon equalities

$$
\begin{aligned}
\bar{u}^{\prime}\left(p^{\prime}\right) \sigma^{\mu \nu} q_{\nu} u(p) & =i \bar{u}^{\prime}\left(p^{\prime}\right)\left(\left(M_{B}+M_{B^{\prime}}\right) \gamma^{\mu}\right. \\
& \left.-\left(p^{\mu}+p^{\prime \mu}\right)\right) u(p)
\end{aligned}
$$

and

$$
\begin{aligned}
\bar{u}^{\prime}\left(p^{\prime}\right) \sigma^{\mu \nu} q_{\nu} \gamma^{5} u(p) & =i \bar{u}^{\prime}\left(p^{\prime}\right)\left(\left(M_{B^{\prime}}-M_{B}\right) \gamma^{\mu} \gamma^{5}\right. \\
& \left.-\left(p^{\mu}+p^{\prime \mu}\right) \gamma^{5}\right) u(p),
\end{aligned}
$$

we can write (4) and (5) as

$$
\begin{aligned}
\left\langle B^{\prime}\left(p^{\prime}\right)\right| & J_{V}^{\mu}|B(p)\rangle \\
& =\bar{u}^{\prime}\left(p^{\prime}\right)\left(\left(f_{1}\left(q^{2}\right)+f_{2}\left(q^{2}\right)\right) \gamma^{\mu}\right. \\
& \left.-\frac{f_{2}\left(q^{2}\right)}{M_{B}+M_{B^{\prime}}}\left(p^{\mu}+p^{\prime \mu}\right)+\frac{f_{3}\left(q^{2}\right)}{M_{B}+M_{B^{\prime}}} q^{\mu}\right) u(p)
\end{aligned}
$$

and

$$
\begin{aligned}
\left\langle B^{\prime}\left(p^{\prime}\right)\right| J_{A}^{\mu} & |B(p)\rangle \\
& =\bar{u}^{\prime}\left(p^{\prime}\right)\left(\left(g_{1}\left(q^{2}\right)+\frac{M_{B^{\prime}}-M_{B}}{M_{B}+M_{B^{\prime}}} g_{2}\left(q^{2}\right)\right) \gamma^{\mu} \gamma^{5}\right. \\
& -\frac{g_{2}\left(q^{2}\right)}{M_{B}+M_{B^{\prime}}}\left(p^{\mu}+p^{\prime \mu}\right) \gamma^{5} \\
& \left.+\frac{g_{3}\left(q^{2}\right)}{M_{B}+M_{B^{\prime}}} q^{\mu} \gamma^{5}\right) u(p) .
\end{aligned}
$$

In the Breit-frame, i.e. the Lorentz-frame, where $\mathbf{p}=$ $-\mathbf{p}^{\prime}=\frac{1}{2} \mathbf{q}$, we obtain in the non-relativistic limit $\left(\mathbf{q}^{2} \ll\right.$ $\left.M_{B}^{2}, M_{B^{\prime}}^{2}\right)$

$$
\begin{aligned}
\left\langle B^{\prime}\left|J_{V}^{0}\right| B\right\rangle & =N_{B} N_{B^{\prime}}^{*} \chi^{\prime \dagger}\left[f_{1}\left(q^{2}\right)+\frac{M_{B}-M_{B^{\prime}}}{M_{B}+M_{B^{\prime}}} f_{3}\left(q^{2}\right)\right] \chi \\
\left\langle B^{\prime}\left|J_{V}^{i}\right| B\right\rangle & =N_{B} N_{B^{\prime}}^{*} \chi^{\prime \dagger}\left[\left(\frac{1}{M_{B}+M_{B^{\prime}}} f_{3}\left(q^{2}\right)\right.\right. \\
& \left.-\frac{M_{B}-M_{B^{\prime}}}{4 M_{B} M_{B^{\prime}}}\left(f_{1}\left(q^{2}\right)+f_{2}\left(q^{2}\right)\right)\right) q^{i} \\
& \left.+i \epsilon^{i j k} \frac{M_{B}+M_{B^{\prime}}}{4 M_{B} M_{B^{\prime}}}\left(f_{1}\left(q^{2}\right)+f_{2}\left(q^{2}\right)\right) q^{j} \sigma^{k}\right] \chi
\end{aligned}
$$

for the vector current and

$$
\begin{aligned}
\left\langle B^{\prime}\left|J_{A}^{0}\right| B\right\rangle & =N_{B} N_{B^{\prime}}^{*} \chi^{\prime \dagger}\left[\frac{M_{B}-M_{B^{\prime}}}{4 M_{B} M_{B^{\prime}}}\left(g_{3}\left(q^{2}\right)-g_{1}\left(q^{2}\right)\right)\right. \\
& \left.-\frac{1}{M_{B}+M_{B^{\prime}}} g_{2}\left(q^{2}\right)\right] \boldsymbol{\sigma} \cdot \mathbf{q} \chi \\
\left\langle B^{\prime}\left|J_{A}^{i}\right| B\right\rangle & =N_{B} N_{B^{\prime}}^{*} \chi^{\prime \dagger}\left[\left(g_{1}\left(q^{2}\right)+\frac{M_{B^{\prime}}-M_{B}}{M_{B}+M_{B^{\prime}}} g_{2}\left(q^{2}\right)\right) \sigma^{i}\right. \\
& +\frac{1}{4 M_{B} M_{B^{\prime}}}\left(g_{3}\left(q^{2}\right)-\frac{1}{2}\left(g_{1}\left(q^{2}\right)\right.\right. \\
& \left.\left.\left.+\frac{M_{B^{\prime}}-M_{B}}{M_{B}+M_{B^{\prime}}} g_{2}\left(q^{2}\right)\right)\right) q^{i} \boldsymbol{\sigma} \cdot \mathbf{q}\right] \chi
\end{aligned}
$$


for the axial-vector current, where $N_{B}\left(N_{B^{\prime}}\right)$ and $\chi\left(\chi^{\prime}\right)$ are a normalization factor and two-component non-relativistic Pauli spinors of the initial (final) baryon state, respectively. We next introduce a set of auxiliary functions according to the following definitions

$$
\begin{aligned}
& \left\langle B^{\prime}\left|J_{V}^{0}\right| B\right\rangle \equiv N_{B} N_{B^{\prime}}^{*} \chi^{\prime \dagger} v_{0} \chi \\
& \left\langle B^{\prime}\left|J_{V}^{i}\right| B\right\rangle \equiv N_{B} N_{B^{\prime}}^{*} \chi^{\prime \dagger}\left(v_{V} q^{i}+i \epsilon^{i j k} v_{A} q^{j} \sigma^{k}\right) \chi \\
& \left\langle B^{\prime}\left|J_{A}^{0}\right| B\right\rangle \equiv N_{B} N_{B^{\prime}}^{*} \chi^{\prime \dagger} a_{0} \boldsymbol{\sigma} \cdot \mathbf{q} \chi \\
& \left\langle B^{\prime}\left|J_{A}^{i}\right| B\right\rangle \equiv N_{B} N_{B^{\prime}}^{*} \chi^{\prime \dagger}\left(a_{S} \sigma^{i}+a_{T} q^{i} \boldsymbol{\sigma} \cdot \mathbf{q}\right) \chi .
\end{aligned}
$$

The functions $v_{0}, v_{V}, v_{A}, a_{0}, a_{S}$, and $a_{T}$ are so called generalized Sachs form factors. The structure of (13) (16) can be deduced from rotational and parity invariance. We also introduce the mass parameters $\Delta \equiv M_{B}-M_{B^{\prime}}$ and $\Sigma \equiv M_{B}+M_{B^{\prime}}$. Identifying the vector functions, we obtain at $\mathbf{q}^{2} \approx 0$

$$
\begin{aligned}
v_{0} & =f_{1}+\frac{\Delta}{\Sigma} f_{3} \\
v_{V} & =-\frac{\Delta}{\Sigma^{2}-\Delta^{2}}\left(f_{1}+f_{2}\right)+\frac{1}{\Sigma} f_{3} \\
v_{A} & =\frac{\Sigma}{\Sigma^{2}-\Delta^{2}}\left(f_{1}+f_{2}\right)
\end{aligned}
$$

and solving these equations for $f_{i}$, where $i=1,2,3$, we get

$$
\begin{aligned}
& f_{1}=v_{0}-\Delta v_{V}-\frac{\Delta^{2}}{\Sigma} v_{A} \\
& f_{2}=-v_{0}+\Delta v_{V}+\Sigma v_{A} \\
& f_{3}=\Sigma v_{V}+\Delta v_{A},
\end{aligned}
$$

at $\mathbf{q}^{2}=0$, which corresponds to $q^{2}=\Delta^{2}$. Similarly, for the axial-vector functions, we obtain at $\mathbf{q}^{2} \approx 0$

$$
\begin{aligned}
& a_{0}=\frac{\Delta}{\Sigma^{2}-\Delta^{2}}\left(g_{3}-g_{1}\right)-\frac{1}{\Sigma} g_{2} \\
& a_{S}=g_{1}-\frac{\Delta}{\Sigma} g_{2} \\
& a_{T}=\frac{1}{\Sigma^{2}-\Delta^{2}}\left(g_{3}-\frac{1}{2}\left(g_{1}-\frac{\Delta}{\Sigma} g_{2}\right)\right)
\end{aligned}
$$

and solving these equations for $g_{i}$, where $i=1,2,3$, we get

$$
\begin{aligned}
g_{1} & =\frac{\Sigma^{2}-\Delta^{2}}{\Sigma^{2}}\left(-\Delta a_{0}+\frac{1}{\Sigma^{2}-\Delta^{2}}\left(\Sigma^{2}-\frac{\Delta^{2}}{2}\right) a_{S}\right. \\
& \left.+\Delta^{2} a_{T}\right) \\
g_{2} & =-\frac{1}{\Sigma}\left(\left(\Sigma^{2}-\Delta^{2}\right) a_{0}+\frac{\Delta}{2} a_{S}\right. \\
& \left.-\left(\Sigma^{2}-\Delta^{2}\right) \Delta a_{T}\right) \\
g_{3} & =\frac{1}{2} a_{S}+\left(\Sigma^{2}-\Delta^{2}\right) a_{T}
\end{aligned}
$$

at $\mathbf{q}^{2}=0$, which corresponds to $q^{2}=\Delta^{2}$. It is important to also keep $f_{3}$ and $g_{3}$ non-zero in order to correctly invert the $v$ 's to the $f$ 's and the $a$ 's to the $g$ 's $[9]$. As mentioned, the $f$ 's and $g$ 's are true Lorentz scalar functions, whereas the generalized Sachs form factors are not. The relations between the $f$ 's and $v$ 's and the $g$ 's and $a$ 's thus depend on the Lorentz-frame in which the calculations are performed, and therefore, all calculations must be performed in the same Lorentz-frame. We have made our calculations in the Breit-frame (which is a good frame (7]), in the nonrelativistic limit.

\subsection{The chiral quark model weak form factors}

Next, we calculate the generalized Sachs form factors in the $\chi \mathrm{QM}$ to linear order in the symmetry breaking. In the $\chi \mathrm{QM}$ the form factors at quark-level are $f_{1}^{q}=1, f_{2}^{q}=0$, $f_{3}^{q}=0, g_{1}^{q}=g_{a}, g_{2}^{q}=0$, and $g_{3}^{q} \neq 0$, since, to lowest order, the $\chi \mathrm{QM}$ vector current is

$$
J_{V, q q^{\prime}}^{\mu}=\bar{\psi}_{q^{\prime}} \gamma^{\mu} \psi_{q}
$$

and the $\chi \mathrm{QM}$ axial-vector current is

$$
J_{A, q q^{\prime}}^{\mu}=g_{a} \bar{\psi}_{q^{\prime}} \gamma^{\mu} \gamma^{5} \psi_{q}-f_{\Phi} \partial^{\mu} \Phi_{q q^{\prime}},
$$

where $g_{a}$ is the quark axial-vector current coupling constant, and $\psi_{q}, q=u, d, s$, are Dirac spinors. The parameter $g_{a}$ was introduced by Manohar and Georgi [11] as a possible "matching parameter" for the $\chi \mathrm{QM}$ Lagrangian after spontaneous symmetry breaking. Later on, we will argue that it should be possible to put $g_{a}=1$, but for the moment we will keep this parameter free.

The term $-f_{\Phi} \partial^{\mu} \Phi_{q q^{\prime}}$ in the axial-vector current (30) appears because of the presence of GBs in the $\chi \mathrm{QM}$. Here $f_{\Phi}$ is the pseudoscalar decay constant and $\Phi_{q q^{\prime}}$ is the pseudoscalar field given by

$$
\Phi=\left(\Phi_{q q^{\prime}}\right)=\left(\begin{array}{ccc}
\frac{\pi^{0}}{\sqrt{2}}+\frac{\eta}{\sqrt{6}} & \pi^{+} & K^{+} \\
\pi^{-} & -\frac{\pi^{0}}{\sqrt{2}}+\frac{\eta}{\sqrt{6}} & K^{0} \\
K^{-} & \bar{K}^{0} & -\frac{2 \eta}{\sqrt{6}}
\end{array}\right) .
$$

The effective Lagrangian for the quark-GB coupling is

$$
\mathcal{L}_{q q^{\prime}}=i g_{8} \bar{\psi}_{q^{\prime}} \Phi_{q^{\prime} q} \gamma^{5} \psi_{q},
$$

where $g_{8} \equiv g_{a}\left(m_{q}+m_{q^{\prime}}\right) / f_{\Phi}$.

In addition to the octet GBs there is also an $\mathrm{SU}(3)$ singlet of $\eta^{\prime}$ bosons. These are coupled to the quarks with a different strength, since the theory would otherwise be $\mathrm{U}(3)$ symmetric, something that does not agree with the measurements of the flavor asymmetry measured by the NMC (New Muon Collaboration) 19,20 in DIS and the NA51 Collaboration 21] in Drell-Yan production. The symmetry breaking SU(3) scalar interaction has the form $\mathcal{L}^{\prime}{ }_{q q}=i g_{0} \frac{1}{\sqrt{3}} \bar{\psi}_{q} \eta^{\prime} \gamma^{5} \psi_{q}$, where $g_{0}$ is the coupling constant for the $\eta^{\prime}$ bosons. 
The effect of this coupling is that the emission of the GBs will in general flip the spin of the quarks. The interaction of the GBs is weak enough to be treated by perturbation theory. This means that on long enough time scales for the low energy parameters to develop we have

$$
\begin{aligned}
& u^{\uparrow} \rightleftharpoons\left(d^{\downarrow}+\pi^{+}\right)+\left(s^{\downarrow}+K^{+}\right)+\left(u^{\downarrow}+\pi^{0}, \eta, \eta^{\prime}\right), \\
& d^{\uparrow} \rightleftharpoons\left(u^{\downarrow}+\pi^{-}\right)+\left(s^{\downarrow}+K^{0}\right)+\left(d^{\downarrow}+\pi^{0}, \eta, \eta^{\prime}\right), \\
& s^{\uparrow} \rightleftharpoons\left(u^{\downarrow}+K^{-}\right)+\left(d^{\downarrow}+K^{0}\right)+\left(s^{\downarrow}+\eta, \eta^{\prime}\right) .
\end{aligned}
$$

The probability of transforming a quark with with spin up by one interaction is given by

$$
\begin{aligned}
\left|\psi\left(u^{\uparrow}\right)\right|^{2} & =\frac{a}{3}\left(2+\zeta^{2}\right) \hat{u}^{\downarrow}+a \hat{d}^{\downarrow}+a \hat{s}^{\downarrow}, \\
\left|\psi\left(d^{\uparrow}\right)\right|^{2} & =a \hat{u}^{\downarrow}+\frac{a}{3}\left(2+\zeta^{2}\right) \hat{d}^{\downarrow}+a \hat{s}^{\downarrow}, \\
\left|\psi\left(s^{\uparrow}\right)\right|^{2} & =a \hat{u}^{\downarrow}+a \hat{d}^{\downarrow}+\frac{a}{3}\left(2+\zeta^{2}\right) \hat{s}^{\downarrow},
\end{aligned}
$$

where $\zeta \equiv g_{0} / g_{8}$ and the coefficient of the $\hat{q}^{\downarrow}$, where $q=$ $u, d, s$, should be interpreted as the probability of creating this quark with spin down by emitting a GB from a quark with spin up. The parameter $a$ measures the probability of emission of a GB from a quark. The total probability of $\mathrm{GB}$ emission is $a\left(8+\zeta^{2}\right) / 3$.

In Fig. 1, diagrams (a) and (b) illustrate the two terms in $(30)$. For $\Delta S=0$ transitions $(d \rightarrow u), \Phi_{d u}=\pi^{-}$, and for $\Delta S=1$ transitions $(s \rightarrow u), \Phi_{s u}=K^{-}$. The second term in the axial-vector current will lead to a non-zero pseudoscalar term (see (41) below), i.e. $g_{3}^{q} \neq 0$. The diagrams (c) - (f) in the same Figure illustrate the emission of GBs that can depolarize the quarks and can even change their flavors.

Spontaneous symmetry breaking in the $\chi \mathrm{QM}$ will give the mass $m_{q}$ to the $q$ quark and the mass $m_{\Phi}$ to the pseudoscalar field and the divergence of the axial-vector current in (30) will be $\partial_{\mu} J_{A, q q^{\prime}}^{\mu}=f_{\Phi} m_{\Phi}^{2} \Phi_{q q^{\prime}}$. Using the Dirac equation for the quarks on the divergence of the quark part of the axial-vector current, one obtains

$$
\left(\square+m_{\Phi}^{2}\right) \Phi_{q q^{\prime}}=i \frac{m_{q}+m_{q^{\prime}}}{f_{\Phi}} g_{a} \bar{\psi}_{q^{\prime}} \gamma^{5} \psi_{q} .
$$

The induced pseudoscalar part of the quark axial-vector current matrix element for the $q \rightarrow q^{\prime}+l^{-}+\bar{\nu}_{l}$ decay is defined as

$$
\begin{aligned}
\left\langle q^{\prime}\left|-f_{\Phi} \partial^{\mu} \Phi_{q q^{\prime}}\right| q\right\rangle & \equiv \bar{u}^{\prime}\left(\frac{g_{3}^{q}}{m_{q}+m_{q^{\prime}}} q^{\mu} \gamma^{5}\right) u \\
& =\frac{g_{3}^{q}}{m_{q}+m_{q^{\prime}}} q^{\mu} \bar{u}^{\prime} \gamma^{5} u
\end{aligned}
$$

Going over to momentum space, we can solve (39) for $\Phi_{q q^{\prime}}$ and insert into $\left\langle q^{\prime}\left|-f_{\Phi} \partial^{\mu} \Phi_{q q^{\prime}}\right| q\right\rangle$, to obtain

$$
\begin{aligned}
\left\langle q^{\prime}\left|-f_{\Phi} \partial^{\mu} \Phi_{q q^{\prime}}\right| q\right\rangle & =\frac{m_{q}+m_{q^{\prime}}}{q^{2}-m_{\Phi}^{2}} g_{a} q^{\mu}\left\langle q^{\prime}\left|\bar{\psi}_{q^{\prime}} \gamma^{5} \psi_{q}\right| q\right\rangle \\
& =\frac{m_{q}+m_{q^{\prime}}}{q^{2}-m_{\Phi}^{2}} g_{a} q^{\mu} \bar{u}^{\prime} \gamma^{5} u
\end{aligned}
$$

This equation corresponds to diagram (b) in Fig. 11. Identifying (40) and (41), we find that

$$
g_{3}^{q}=\frac{\sigma^{2}}{\Delta^{2}-m_{\Phi}^{2}} g_{a}
$$

at $q^{2}=\Delta^{2}$. Note that $(42)$ is $\mathcal{O}\left(f_{\Phi}^{0}\right)$, thus the two diagrams (a) and (b) in Fig. 1 are of the same order in $f_{\Phi}$.

We will now make the assumption that the kinetic energy of the constituent quarks is small enough to allow us to use the static approximation for them. The advantage of this is that the results will be less model dependent than by using bound state model wave functions. The disadvantage is of course that it might be too rough an approximation. On the other hand, we should understand these calculations to be done at the same level of approximation for both the magnetic moments and the weak form factors, since the effective quark parameters can then be used to relate these observables to each other. If we change the model for one of these sets of observables, this would not be possible.

Using the equivalents of (17) - (19) and (23) - (25), we obtain at quark-level

$$
\begin{aligned}
v_{0}^{q} & =1 \\
v_{V}^{q} & =-\frac{\delta}{\sigma^{2}-\delta^{2}} \\
v_{A}^{q} & =\frac{\sigma}{\sigma^{2}-\delta^{2}}
\end{aligned}
$$

and

$$
\begin{aligned}
a_{0}^{q} & =\frac{\delta}{\sigma^{2}-\delta^{2}}\left(g_{3}^{q}-g_{a}\right) \\
a_{S}^{q} & =g_{a} \\
a_{T}^{q} & =\frac{1}{\sigma^{2}-\delta^{2}}\left(g_{3}^{q}-\frac{g_{a}}{2}\right),
\end{aligned}
$$

where $\delta \equiv m_{q}-m_{q^{\prime}}, \sigma \equiv m_{q}+m_{q^{\prime}}$, and $g_{3}^{q}$ is the induced pseudoscalar form factor at quark-level.

The quark current operators (29) and (30) will be sandwiched between baryon state vectors with (total) spin up in both the initial and the final states. In the nonrelativistic limit, the current operators then act additively on the three quarks in the baryons. We will therefore use the Sachs form factors for the quark currents, and identify the corresponding Sachs form factors for the baryons by their kinematic structure.

The flavor changing quark transitions can be conveniently expressed by means of the $\lambda_{q q^{\prime}}$ matrices, which are combinations of $\mathrm{SU}(3)$ Gell-Mann matrices. For the $\Delta S=0$ decays $\left(\lambda_{d u}\right)$ and the $\Delta S=1$ decays $\left(\lambda_{s u}\right)$, we have

$$
\lambda_{d u}=\left(\begin{array}{lll}
0 & 1 & 0 \\
0 & 0 & 0 \\
0 & 0 & 0
\end{array}\right) \quad \text { and } \quad \lambda_{s u}=\left(\begin{array}{lll}
0 & 0 & 1 \\
0 & 0 & 0 \\
0 & 0 & 0
\end{array}\right)
$$

The operators to be sandwiched between the baryonic quark model states, to obtain the Sachs form factors, are 
therefore

$$
\begin{aligned}
v_{0, q q^{\prime}} & =\lambda_{q q^{\prime}} \otimes 1 \\
v_{V, q q^{\prime}} & =-\frac{\delta}{\sigma^{2}-\delta^{2}} \lambda_{q q^{\prime}} \otimes 1 \\
v_{A, q q^{\prime}} & =\frac{\sigma}{\sigma^{2}-\delta^{2}} \lambda_{q q^{\prime}} \otimes \sigma^{z}
\end{aligned}
$$

and

$$
\begin{aligned}
& a_{0, q q^{\prime}}=\frac{\delta}{\sigma^{2}-\delta^{2}}\left(g_{3}^{q}-g_{a}\right) \lambda_{q q^{\prime}} \otimes \sigma^{z} \\
& a_{S, q q^{\prime}}=g_{a} \lambda_{q q^{\prime}} \otimes \sigma^{z} \\
& a_{T, q q^{\prime}}=\frac{1}{\sigma^{2}-\delta^{2}}\left(g_{3}^{q}-\frac{g_{a}}{2}\right) \lambda_{q q^{\prime}} \otimes \sigma^{z} .
\end{aligned}
$$

The $\lambda_{q q^{\prime}}$ matrix effectuates the flavor transition and the $\sigma^{z}$ operator measures the spin polarizations of the quarks in the baryons.

In a given type of transition, say $\Delta S=1$, the active quark masses are the same and the spectator quark masses do not enter explicitly in the calculations. Introducing the notation $f_{1}^{\mathrm{QM}} \equiv\left\langle B^{\prime}\left|\lambda_{q q^{\prime}} \otimes 1\right| B\right\rangle$ and $g_{1}^{\mathrm{QM}} \equiv$ $\left\langle B^{\prime}\left|\lambda_{q q^{\prime}} \otimes \sigma^{z}\right| B\right\rangle$, we can identify the $v$ 's in the baryonic matrix element and insert them into (20) - 22), to obtain

$$
\begin{aligned}
& f_{1}=\left(1+\frac{\Delta}{\sigma^{2}-\delta^{2}}\left(\delta-\frac{\sigma}{\Sigma} \Delta \frac{g_{1}^{\mathrm{QM}}}{f_{1}^{\mathrm{QM}}}\right)\right) f_{1}^{\mathrm{QM}}, \\
& f_{2}=\left(\frac{1}{\sigma^{2}-\delta^{2}}\left(\Sigma \sigma \frac{g_{1}^{\mathrm{QM}}}{f_{1}^{\mathrm{QM}}}-\Delta \delta\right)-1\right) f_{1}^{\mathrm{QM}},
\end{aligned}
$$

and

$$
f_{3}=\frac{1}{\sigma^{2}-\delta^{2}}\left(\Delta \sigma \frac{g_{1}^{\mathrm{QM}}}{f_{1}^{\mathrm{QM}}}-\Sigma \delta\right) f_{1}^{\mathrm{QM}} .
$$

In a similar way, we can identify the $a$ 's and insert them into (26) - (28). The result is

$$
\begin{gathered}
g_{1}=\left(1+\frac{\Sigma^{2}-\Delta^{2}}{\Sigma^{2}}\left(\frac{\Delta \delta}{\sigma^{2}-\delta^{2}}-\frac{\Delta^{2}}{2}\left(\frac{1}{\Sigma^{2}-\Delta^{2}}\right.\right.\right. \\
\left.\left.\left.+\frac{1}{\sigma^{2}-\delta^{2}}\right)+\frac{\Delta}{\sigma^{2}-\delta^{2}}(\Delta-\delta) \frac{g_{3}^{q}}{g_{a}}\right)\right) g_{a} g_{1}^{\mathrm{QM}} \\
g_{2}=\frac{1}{\Sigma}\left(\frac{\Sigma^{2}-\Delta^{2}}{\sigma^{2}-\delta^{2}}\left(\delta-\frac{\Delta}{2}\right)-\frac{\Delta}{2}\right. \\
\left.+\frac{\Sigma^{2}-\Delta^{2}}{\sigma^{2}-\delta^{2}}(\Delta-\delta) \frac{g_{3}^{q}}{g_{a}}\right) g_{a} g_{1}^{\mathrm{QM}}
\end{gathered}
$$

and

$$
g_{3}=\left(\frac{1}{2}\left(1-\frac{\Sigma^{2}-\Delta^{2}}{\sigma^{2}-\delta^{2}}\right)+\frac{\Sigma^{2}-\Delta^{2}}{\sigma^{2}-\delta^{2}} \frac{g_{3}^{q}}{g_{a}}\right) g_{a} g_{1}^{\mathrm{QM}}
$$

The weak currents on baryon-level and quark-level have to be calculated in the same reference frame in order to maintain Lorentz invariance of the weak form factors $f_{i}$ and $g_{i}$, where $i=1,2,3$ [7].

The final result will contain a multiplicative factor from the wave function overlap, contributing to the so called wave function mismatch. Actually, this mismatch comes about from two different sources.

The first one is the recoil effect, that for non-relativistic systems is proportional to the matrix element of the spherical Bessel function $j_{0}(\Delta r)$, where $r$ is the radial coordinate. If we consider an expansion in $\delta$ and $\Delta$, we get

$$
j_{0}(\Delta r) \equiv \frac{\sin (\Delta r)}{\Delta r}=1-\frac{1}{6} \Delta^{2} r^{2}+\cdots .
$$

The contribution from this term that is different from 1 is therefore $\mathcal{O}\left(\Delta^{2}\right)$. For spherically symmetric wave functions ( $S$-waves), the lowest order relativistic effects in the kinematic terms can also be shown to be $\mathcal{O}\left(\delta^{2}\right)$.

Secondly, we have the contribution from the overlap between two wave functions that have different quark masses. By expanding the wave function in the quark mass difference $\delta$, it can easily be shown that the deviation of this effect from 1 is also $\mathcal{O}\left(\delta^{2}\right)$. Since we are calculating only the linear part of the symmetry breaking in the weak form factors, we will therefore in the following neglect the wave function mismatch.

Define now the parameters $E \equiv \Delta / \Sigma$ and $\epsilon \equiv \delta / \sigma$. If we express (55) - (57) and (58) - (60) in $\Sigma, E, \sigma$, and $\epsilon$ and neglect all terms which are proportional to $E^{2}, \epsilon^{2}$, and $E \epsilon$, we obtain

$$
\begin{aligned}
f_{1} & =f_{1}^{\mathrm{QM}} \\
f_{2} & =\left(\frac{\Sigma}{\sigma} G_{A}-1\right) f_{1}^{\mathrm{QM}} \\
f_{3} & =\frac{\Sigma}{\sigma}\left(E G_{A}-\epsilon\right) f_{1}^{\mathrm{QM}}
\end{aligned}
$$

and

$$
\begin{aligned}
& g_{1}=g_{a} g_{1}^{\mathrm{QM}} \\
& g_{2}=\left(\frac{\Sigma}{\sigma} \epsilon-\frac{1}{2}\left(1+\frac{\Sigma^{2}}{\sigma^{2}}\right) E\right) g_{a} g_{1}^{\mathrm{QM}} \\
& g_{3}=\left(\frac{1}{2}\left(1-\frac{\Sigma^{2}}{\sigma^{2}}\right)+\frac{\Sigma^{2}}{\sigma^{2}} \frac{g_{3}^{q}}{g_{a}}\right) g_{a} g_{1}^{\mathrm{QM}},
\end{aligned}
$$

where $G_{A} \equiv g_{1}^{\mathrm{QM}} / f_{1}^{\mathrm{QM}}$. In this result we have also deleted the term in $g_{2}$ proportional to $g_{3}^{q}$, since it should be absent on physical grounds. The current piece containing the $g_{2}$ form factor is orthogonal to $q_{\mu}$, whereas $g_{3}^{q}$ is proportional to the divergence of the axial-vector current.

We note that the first-class current form factors $f_{1}, f_{2}$, $g_{1}$, and $g_{3}$ only contain terms with even powers of $E$ and $\epsilon$, while the second-class current form factors $f_{3}$ and $g_{2}$ only contain terms with odd powers of $E$ and $\epsilon$. This follows from the Ademollo-Gatto theorem [22,7]. The above expressions for $f_{i}$ and $g_{i}$, where $i=1,2,3$, in (61) - (66) are evaluated at $q^{2}=\Delta^{2}$. 
Using (42), this means that (64) - (66) now can be expressed as

$$
\begin{aligned}
& g_{1}=g_{a} g_{1}^{\mathrm{QM}} \\
& g_{2}=\left(\frac{\Sigma}{\sigma} \epsilon-\frac{1}{2}\left(1+\frac{\Sigma^{2}}{\sigma^{2}}\right) E\right) g_{a} g_{1}^{\mathrm{QM}} \\
& g_{3}=\left(\frac{1}{2}\left(1-\frac{\Sigma^{2}}{\sigma^{2}}\right)+\frac{\Sigma^{2}}{\Delta^{2}-m_{\Phi}^{2}}\right) g_{a} g_{1}^{\mathrm{QM}},
\end{aligned}
$$

in the $\chi \mathrm{QM}$. We will keep the $\Delta^{2}$ term in the denominator in (69), since there is no natural expansion parameter in this case.

The usual way to obtain the values of the form factors $f_{1}$ and $g_{1}$ at $q^{2}=0$ is to use the empirical dipole forms for the $q^{2}$ dependence of these form factors. It is easy to see that the difference between, say, $f_{1}(0)$ and $f_{1}\left(\Delta^{2}\right)$ is $\mathcal{O}\left(\Delta^{2}\right)$. But, since the form factors $f_{1}$ and $g_{1}$ are only valid up to quadratic terms in the mass differences, we will neglect the $q^{2}$ dependence from the empirical dipole forms and put $f_{1}=f_{1}\left(\Delta^{2}\right) \approx f_{1}(0)$ and $g_{1}=g_{1}\left(\Delta^{2}\right) \approx g_{1}(0)$.

The $q^{2}$ dependences of the other form factors $f_{2}, f_{3}, g_{2}$, and $g_{3}$ are also neglected, since these dependences cannot be decided with the current level of experimental precision.

In the $\chi \mathrm{QM}$, the effective quark masses can be determined from the fitted value of $\mu_{d}$, which in the $\chi \mathrm{QM}$ is $\mu_{d} \approx-1.35 \mu_{N}$ 15. Using this value together with the formulas from the magnetic moments, $\mu_{u}=-2 \mu_{d}$, and $\mu_{s}=2 \mu_{d} / 3$ [15], the effective quark masses in the $\chi \mathrm{QM}$ are $m_{u}^{\mathrm{eff}}=m_{d}^{\mathrm{eff}}=m^{\mathrm{eff}} \approx 230 \mathrm{MeV}$ and $m_{s}^{\mathrm{eff}}=3 m^{\mathrm{eff}} / 2 \approx$ $350 \mathrm{MeV}$. For the form factor $g_{3}$ we have used $m_{\pi}=140$ $\mathrm{MeV}$ and $m_{K}=490 \mathrm{MeV}$. This seems to be consistent with the pole in $g_{3}$ coming from $g_{3}^{q}$ being identified with the pion (kaon) pole in $g_{3}$ from dispersion relations.

\subsection{The weak axial-vector form factors}

The weak axial-vector form factors $G_{A}=g_{1}^{\mathrm{QM}} / f_{1}^{\mathrm{QM}}$ can be obtained from the $\mathrm{SU}(6)$ quark model expressions for $f_{1}$ and $g_{1}$ expressed in terms of the parameters $F$ and $D$ [23]. In the $\chi \mathrm{QM}$, the weak axial-vector form factors $G_{A}$ are expressed in the quark spin polarizations of the proton, i.e. $\Delta u, \Delta d$, and $\Delta s$. These spin polarizations differ considerably from the ones in the $\mathrm{SU}(6)$ quark model due to the depolarization of the quark spins by the GBs. The spin polarizations in the $\chi \mathrm{QM}$ are calculated to $\mathcal{O}\left(f_{\Phi}\right)$, i.e. with one GB emission. They are 13]

$$
\begin{aligned}
& \Delta u=\frac{4}{3}-\frac{a}{9}\left(8 \zeta^{2}+37\right), \\
& \Delta d=-\frac{1}{3}+\frac{2 a}{9}\left(\zeta^{2}-1\right) \\
& \Delta s=-a .
\end{aligned}
$$

For values of $\Delta u, \Delta d$, and $\Delta s$ in the $\chi \mathrm{QM}$, see Table 1 . Using the relations $F=\frac{1}{2}(\Delta u-\Delta s)$ and $D=\frac{1}{2}(\Delta u-$
$2 \Delta d+\Delta s)$ [2], we have

$$
\begin{aligned}
G_{A}^{n p} & =\Delta u-\Delta d \\
G_{A}^{\Sigma^{-} \Sigma^{0}} & =\frac{1}{2}(\Delta u-\Delta s) \\
g_{1}^{\mathrm{QM}^{\Sigma^{ \pm}} \Lambda} & =\frac{1}{\sqrt{6}}(\Delta u-2 \Delta d+\Delta s) \\
G_{A}^{\Xi^{-} \Xi^{0}} & =\Delta d-\Delta s
\end{aligned}
$$

for the $\Delta S=0$ decays and

$$
\begin{aligned}
G_{A}^{\Sigma^{-} n} & =\Delta d-\Delta s \\
G_{A}^{\Xi^{-} \Sigma^{0}} & =\Delta u-\Delta d \\
G_{A}^{\Xi^{-}} & =\frac{1}{3}(\Delta u+\Delta d-2 \Delta s) \\
G_{A}^{\Lambda p} & =\frac{1}{3}(2 \Delta u-\Delta d-\Delta s) \\
G_{A}^{\Xi^{0} \Sigma^{+}} & =\Delta u-\Delta d
\end{aligned}
$$

for the $\Delta S=1$ decays.

The $\Sigma^{0} \rightarrow \Sigma^{+}+l^{-}+\bar{\nu}_{l}$ and $\Sigma^{0} \rightarrow p+l^{-}+\bar{\nu}_{l}$ decays cannot be observed, since the electromagnetic decay $\Sigma^{0} \rightarrow \Lambda+\gamma$ is predominant. The corresponding $G_{A}$ 's are therefore not listed above.

The values of the $G_{A}^{B B^{\prime}}$ 's for the $\chi \mathrm{QM}$ are listed in Table2, where for reference also the axial-vector form factors of the NQM are displayed.

The weak axial-vector form factor $g_{A}$ is defined as

$$
g_{A} \equiv \frac{g_{1}}{f_{1}}
$$

For the weak vector form factor $f_{1}$, the $\chi \mathrm{QM}$ gives the same result as the ordinary NQM. The appropriate values can be found in Table 3. We thus obtain the simple result

$$
g_{A}=g_{a} G_{A} .
$$

It has been argued by Weinberg [24, that not only in QCD, but also in the effective Lagrangians, one should expect $g_{a}=1$, since the matrix element algebra of the axial-vector currents between color quark states should be saturated by the single quark state to leading order in $1 / N_{c}$, where $N_{c}$ is the number of colors. This leads to $g_{a}=1$. Compare with the Adler-Weisberger relation, that relates the deviation of $g_{A}^{n p}$ from 1 to the presence of excited intermediate states, like the $\Delta(1232)$ resonance, in the saturation of the sum-rule. The subleading order corrections that come from quark-GB interactions 25] are taken care of by the depolarization of the quark spins due to GB emission above. The renormalization of the axialvector form factor for $g_{A}^{n p}$ from its $\mathrm{SU}(6)$ value of $5 / 3$ to its experimental value of 1.26 should then come entirely from the change in spin polarization due to the GBs, otherwise there is a risk for double counting. This attitude for $g_{a}$ in the $\chi \mathrm{QM}$ has also been taken by other authors 113 , 15, 26, 22, 28] and will be adopted here. See, however, also Ref. 29.

Expressed in terms of matrix elements, the weak axialvector form factors $g_{A}^{B B^{\prime}}$ in the $\chi \mathrm{QM}$ will then equal to $g_{A}^{B B^{\prime}}=G_{A}^{B B^{\prime}}(\Delta u, \Delta d, \Delta s)$ as given above. 


\subsection{The ratio $\rho_{f}$ and the "weak magnetism"}

We next turn to the "weak magnetism" form factor $\rho_{f}$, which is defined as

$$
\rho_{f} \equiv \frac{f_{2}}{f_{1}} .
$$

Inserting (61) and (62) in (84), we obtain

$$
\rho_{f}=\frac{\Sigma}{\sigma} G_{A}-1
$$

The formula above can be transformed into an expression in terms of the magnetic moments of the baryons. For example, for the $n \rightarrow p+l^{-}+\bar{\nu}_{l}$ decay, we can show, using $\mu_{p}=\Delta u \mu_{u}+\Delta d \mu_{d}+\Delta s \mu_{s}$ and the corresponding formula for $\mu_{n}$, that

$$
\begin{aligned}
\rho_{f}^{n p} & =\frac{1}{2}\left(1+\frac{M_{n}}{M_{p}}\right)\left(\mu_{p}-\mu_{n}\right) \frac{1}{\mu_{N}}-1 \\
& \approx\left(\mu_{p}-\mu_{n}\right) \frac{1}{\mu_{N}}-1 .
\end{aligned}
$$

Here we have used the expression $G_{A}^{n p}=\Delta u-\Delta d$ from Subsection 2.3 above and $\mu_{u}=-2 \mu_{d}$. Equation (86) is exactly the conserved vector current (CVC) formula for the $n \rightarrow p+l^{-}+\bar{\nu}_{l}$ decay. Using the $\chi \mathrm{QM}$ values $\mu_{p} \approx 2.67 \mu_{N}$ and $\mu_{n} \approx-1.86 \mu_{N}$ [15], we thus obtain $\rho_{f}^{n p} \approx 3.53$, in agreement with the direct calculation (see Table 1).

The expression for $\rho_{f}$ above is closely related to the corresponding formula for the magnetic moments $\mu_{B}$ of the octet baryons used in earlier studies. In the same approximation as here, we have

$$
f_{2}(0) / f_{1}(0)=\Sigma \mu_{B}-1=\Sigma \sum_{q=u, d, s} \frac{e_{q}}{2 m_{q}} \Delta q-1,
$$

where $e_{q}$ is the quark charge.

When these expressions are fitted to the baryon magnetic moments, the quark masses appear as effective masses, and the parametric dependence of the quark spin polarization $\Delta q$ on the emission probability a of GBs incorporates effects of relativistic corrections and other possible dynamical effects on the magnetic moments [30]. When these effects are taken into account directly, in terms of a changed structure of the currents, the fits become quite bad 28 . At the present time the above treatment is therefore probably the best one can hope for.

\subsection{The ratio $\rho_{g}$ and the weak form factor $g_{P T}$}

The ratio $\rho_{g}$ is defined as

$$
\rho_{g} \equiv \frac{g_{2}}{g_{1}}
$$

Thus $\rho_{g}$ is obtained by dividing (68) by 67

$$
\rho_{g}=\frac{\Sigma}{\sigma} \epsilon-\frac{1}{2}\left(1+\frac{\Sigma^{2}}{\sigma^{2}}\right) E .
$$

The ratio $\rho_{g}$ depends only on the masses of the quarks $q$, $q^{\prime}$ and the baryons $B, B^{\prime}$, and not on $g_{a}$.

The weak induced pseudotensor form factor $g_{P T}$ is defined as

$$
g_{P T} \equiv \frac{g_{2}}{f_{1}}
$$

We then have

$$
g_{P T}=\left(\frac{\Sigma}{\sigma} \epsilon-\frac{1}{2}\left(1+\frac{\Sigma^{2}}{\sigma^{2}}\right) E\right) G_{A} .
$$

Using the ratio $\rho_{g} \equiv g_{2} / g_{1}$, we can relate the form factor $g_{P T}$ to the form factor $g_{A}$ according to

$$
g_{P T}=\frac{g_{2}}{f_{1}}=\frac{g_{2}}{g_{1}} \frac{g_{1}}{f_{1}}=\rho_{g} g_{A} .
$$

The matrix elements of the weak induced pseudotensor form factor $g_{P T}$ are thus given by

$$
g_{P T}^{B B^{\prime}}=\rho_{g}^{B B^{\prime}} G_{A}^{B B^{\prime}} .
$$

Since different signs for $\rho_{g}$ are obtained in different models (see Table 5) we would like to see if we can understand this feature from our estimate. Inspection of (89) shows that its sign will depend upon a balance between the term proportional to $\epsilon$ and the one proportional to $E$.

For the $\Delta S=0 \Sigma^{ \pm} \rightarrow \Lambda$ transitions $\epsilon=0$ so $\rho_{g}$ is negative. This is consistent with the values presented by all authors and affirms that the same sign convention is used.

For the $\Delta S=1$ transitions $\epsilon \neq 0$ and the situation depends on the balance between the terms. Since $(1+$ $\left.\Sigma^{2} / \sigma^{2}\right) \approx \Sigma^{2} / \sigma^{2}$ for these decays, the sign of $\rho_{g}$ depends on the sign of $\delta-\Delta / 2$. This value depends evidently upon the models used. In our case the sign is negative for the $\Sigma^{-} \rightarrow n$ transition and positive for the others.

A similar remark applies to the form factor ratio $f_{3} / f_{1}$. Its sign is also dependent upon a balance between two terms. For the $\Delta S=0 \Sigma^{ \pm} \rightarrow \Lambda$ transitions, where $\epsilon=0$, we have $f_{3}=g_{1}^{\mathrm{QM}} \Delta / \sigma$, which is positive. For the $\Delta S=1$ transitions we can only say for sure that it must be negative for decays with negative $G_{A}$. Since it is not possible at present to measure $f_{3}$ we will not study it any further.

Also the form factor $g_{3}$ is not possible to measure at present, although the pole term makes it quite large.

In our calculations we have $\delta \approx 120 \mathrm{MeV}$ and $\epsilon \approx 0.20$ for the $\Delta S=1$ transitions. This means that some of the form factors should be considered as estimates rather than calculations. Nevertheless, such estimates are often much better than one would expect. In particular, as has been mentioned above, the ratios $\Delta q / m_{q}$, where $\Delta q$ is the spin polarization and $m_{q}$ the effective mass of a quark with flavor $q$, are well determined from the magnetic moment calculations, and should reproduce the different weak form factors well. In our opinion, the over all performance of the $\chi \mathrm{QM}$ is quite good, and with one possible exception, it reproduces the experimental data. 


\section{Numerical results}

\subsection{Experimental values of the weak axial-vector form factors}

The measured weak axial-vector form factor, $g_{A}^{\exp }$, is often a superposition of the theoretical weak axial-vector form factor $g_{A}$ and the theoretical weak induced pseudotensor form factor $g_{P T}$, since one assumes that the form factor $g_{2}$ is zero in the analysis of data. Thus from the Gordon equality (6), one gets

$$
g_{A}^{\exp }=g_{A}-E g_{P T},
$$

where $E$ is given by

$$
E \equiv \frac{M_{B}-M_{B^{\prime}}}{M_{B}+M_{B^{\prime}}}
$$

As a quasi-experimental value for $g_{A}$ one could take the value obtained by solving (94) for $g_{A}$ and inserting our theoretical prediction for $g_{P T}$. Thus,

$$
g_{A}^{\text {quasi }} \equiv g_{A}^{\text {exp }}+E g_{P T}^{\text {theory }} .
$$

However, since $E$ is quite small, $E \leq 0.12$, and $g_{P T}$ is also small, the term $E g_{P T}$ is negligible in our approximation. This is consistent with $E g_{P T}$ being $\mathcal{O}\left(E^{2}, E \epsilon\right)$. The experimental values of the weak axial-vector form factors $g_{A}^{B B^{\prime}}$ axp are presented in Table 6 .

For the $\Sigma^{-} \rightarrow n+l^{-}+\bar{\nu}_{l}$ decay, we have $E^{\Sigma^{-} n} \approx$ 0.12 according to (95). Hsueh et al. [3] have measured $g_{A}^{\Sigma^{-} n^{\exp }}=-0.327 \pm 0.007 \pm 0.019$ in a single parameter fit which corresponds to (94), and also independently $g_{A}^{\Sigma^{-} n}=$ $-0.20 \pm 0.08$ and $g_{P T}^{\Sigma^{-}} n^{\text {Hsueh }}=0.56 \pm 0.37$. Hsueh et al. use a definition of $g_{P T}$ different from ours, and the definitions are related to each other by the formula

$$
g_{P T}^{\Sigma^{-} n}=\left(1+\frac{M_{n}}{M_{\Sigma^{-}}}\right) g_{P T}^{\Sigma^{-}{ }^{H} \mathrm{Hsueh}} .
$$

Equation (97) gives $g_{P T}^{\Sigma^{-} n}=1.00 \pm 0.66$. Using the definition of $\rho_{g}$, we now get $\rho_{g}^{\Sigma^{-} n}=g_{P T}^{\Sigma^{-} n} / g_{A}^{\Sigma^{-} n}=-5.0 \pm 3.9$. None of the presented models in the tables are able to reproduce this value.

\subsection{Discussion}

In Table 6 we present the $\chi \mathrm{QM}$ values for the $g_{A}$ 's. The value of $g_{A}^{n p}$ is slightly low in the $\chi \mathrm{QM}$. This indicates that the theoretical values are still maybe only within about $10 \%$ of the experimental ones. It is also possible that a fine tuning of the value for the parameter that measures the strength of the GB emission could bring the value up. Nevertheless, the agreement between the experimental values and the model is quite encouraging and represents a clear improvement over the NQM values.

In the $\mathrm{SU}(6)$ model, the value $g_{A}^{n p}=5 / 3$ is related to the value of $g_{A}$ for the transition $p \rightarrow \Delta^{++}$, when the axial-vector matrix element algebra is saturated with the octet and decuplet [31.

The improvement of $g_{A}^{n p}$ in the $\chi \mathrm{QM}$ is due to the effect of the GB emission from the quarks before or after the weak interaction. This changes the matrix element algebra of the axial-vector currents that fixes the value of $g_{A}$, since both before and after the interaction the quark amplitude in the baryonic states are not in pure $\mathrm{SU}(6)$ representations, but rather in a mixture of such states, not only of different spins, but also of different flavors.

When it comes to the other form factors the situation is as follows.

For the $\rho_{f}$ ratios there are more experimental data than for the $\rho_{g}$ ratios. Let us therefore consider Table 1 . All values obtained for the $\rho_{f}$ 's in the $\chi \mathrm{QM}$ lie within the experimental errors, where experimental data exist. (The experimental results have large errors, though.) The CVC values listed are in a way half experimental results, since they use the measured values of the anomalous magnetic moments for the nucleons as input data to calculate these values. All calculated values for the $\chi \mathrm{QM}$ have the same sign as the CVC values and they are also close in magnitude. This is of course related to the fact that the form factors are calculated in the same approximation as the magnetic moments in earlier studies, and the parameters from these calculations are used here. For some cases we can see that $\rho_{f}(\chi \mathrm{QM}) \approx \rho_{f}(\mathrm{CVC})$, as for the neutron decay. For other decays the $\rho_{f}$ 's of the $\chi \mathrm{QM}$ incorporate effects of vector current non-conservation due to the mass differences between the isomultiplets.

Let us then consider Table f. Unfortunately, only one of the $\rho_{g}$ 's, namely $\rho_{g}^{\Sigma^{-} n}$, has been measured experimentally. As mentioned before, this was done by Hsueh et al. They found $\rho_{g}^{\Sigma^{-} n}=-5.0 \pm 3.9$ (in our conventions). Theoretically, our estimate gives the value -0.143 in the $\chi \mathrm{QM}$, and this is not in agreement with the experimental value. However, also the values of all other models are outside the experimental range. Taken at face value, the result for $\rho_{g}^{\Sigma^{-} n}$ as measured by Hsueh et al. [3] would tend to favor models with negative values for the $\rho_{g}$. However, one should perhaps await further measurements before taking a stand, since the error is quite large, and one more standard deviation would allow for models with positive $\rho_{g}$.

For the other $\rho_{g}$ 's with $\Delta S=1$, we can only compare our predictions with previous model calculations. We get a positive sign for these $\rho_{g}$ 's in agreement with the MIT and $\chi \mathrm{QSM}_{\mathrm{br}}$ models. The other models have negative signs for the $\rho_{g}$ 's.

Finally, we present in Table 6 model estimates for the $g_{P T}$ 's calculated using the values of $\rho_{g}$ and $g_{A}$ presented above. The $\chi \mathrm{QM}$ value for $g_{P T}^{\Sigma^{-}}$, which is the only measured form factor, is too small compared to the experiment.

The over all picture of our theoretical estimates for the $\chi \mathrm{QM}$ are, apart from the measured value of the form factor $g_{P T}$ and the value of $\rho_{g}$ for the $\Sigma^{-} \rightarrow n$ transition, in good agreement with the existing experimental data. 


\section{Summary and conclusions}

We have presented a study of the baryonic weak vector and axial-vector form factors in the spirit of the chiral quark model. The results are presented in Tables 3- 6, and the over all agreement with existing data is satisfactory and represents a clear improvement with respect to the nonrelativistic quark model.

The experimental axial-vector form factors, corrected for the possible non-zero values of $g_{P T}$, are of importance in e.g. the analysis of the quark spin polarizations of the nucleon. Our study supports the assumption that these form factors are small. The second-class form factors $f_{3}$ and $g_{2}$ are also highly model dependent.

The present investigation has used the $\mathrm{SU}(3)$ symmetric coupling in the chiral quark model and the static approximation for the quarks as a first approximation. A natural improvement would be to incorporate lowest order non-static effects and further SU(3) symmetry breaking effects 26, 32, to obtain better agreement with experimental data. In particular, we expect that this would lead to a closer agreement with the $\rho_{f}$ ratios from the conserved vector current theory, since symmetry breaking can better account for the octet baryon magnetic moments [15]. $\mathrm{SU}(3)$ symmetry breaking also leads to better agreement for $g_{A}^{n p} 15,26,32$.

Finally, we think that it would be quite interesting to have more measurements of $\rho_{g}$ for various transitions, since this parameter might help to distinguish between different models.

Acknowledgements. We would like to thank Johan Linde for useful comments on the manuscript. This work was supported by the Swedish Natural Science Research Council (NFR), Contract No. F-AA/FU03281-310. Support for this work was also provided by the Engineer Ernst Johnson Foundation (T.O.).

\section{References}

1. European Muon Collaboration, J. Ashman et al., Phys. Lett. B 206, 364 (1988); Nucl. Phys. B328, 1 (1989)

2. F.E. Close, The Nucleon Spin Crisis Bible, RAL-93-034, 1993 (unpublished); F.E. Close and R.G. Roberts, Phys. Lett. B 316, 165 (1993)

3. S.Y. Hsueh et al., Phys. Rev. D 38, 2056 (1988)

4. B.H. Kellett, Phys. Rev. D 10, 2269 (1974)

5. J.F. Donoghue and B.R. Holstein, Phys. Rev. D 25, 206 (1982)

6. J.O. Eeg and Ø. Lie-Svendsen, Z. Phys. C 27, 119 (1985)

7. J.O. Eeg, H. Høgaasen, and Ø. Lie-Svendsen, Z. Phys. C 31, 443 (1986)

8. Ø. Lie-Svendsen and H. Høgaasen, Z. Phys. C 35, 239 (1987)

9. L.J. Carson, R.J. Oakes, and C.R. Willcox, Phys. Rev. D 37, 3197 (1988)

10. H.-C. Kim, M.V. Polyakov, M. Praszałowicz, and K. Goeke, Phys. Rev. D 57, 299 (1998)

11. A. Manohar and H. Georgi, Nucl. Phys. B234, 189 (1984)

12. E.J. Eichten, I. Hinchliffe, and C. Quigg, Phys. Rev. D 45, 2269 (1992)
13. T.P. Cheng and L.-F. Li, Phys. Rev. Lett. 74, 2872 (1995)

14. T.P. Cheng and L.-F. Li, in Proceedings of the International Europhysics Conference on High Energy Physics (HEP 95), Brussels, Belgium, 1995, edited by J. Lemonne et al. (World Scientific, Singapore 1996), hep-ph/9510236

15. J. Linde, T. Ohlsson, and H. Snellman, Phys. Rev. D 57, 452 (1998)

16. J. Linde, T. Ohlsson, and H. Snellman, Phys. Rev. D 57, 5916 (1998)

17. J.F. Donoghue, E. Golowich, and B.R. Holstein, Dynamics of the Standard Model (Cambridge, Cambridge 1992)

18. S. Weinberg, Phys. Rev. 112, 1375 (1958)

19. New Muon Collaboration, P. Amaudruz et al., Phys. Rev. Lett. 66, 2712 (1991)

20. New Muon Collaboration, M. Arneodo et al., Phys. Rev. D 50, R1 (1994)

21. NA51 Collaboration, A. Baldit et al., Phys. Lett. B 332, 244 (1994)

22. M. Ademollo and R. Gatto, Phys. Rev. Lett. 13, 264 (1964)

23. P. Renton, Electroweak Interactions - An Introduction to the Physics of Quarks 85 Leptons (Cambridge, Cambridge 1990)

24. S. Weinberg, Phys. Rev. Lett. 65, 1181 (1990)

25. S. Weinberg, Phys. Rev. Lett. 67, 3473 (1991)

26. T.P. Cheng and L.-F. Li, Phys. Rev. D 57, 344 (1998)

27. H.J. Weber, X. Song, and M. Kirchbach, Mod. Phys. Lett. A 12, 729 (1997)

28. H.J. Weber and K. Bodoor, Int. J. Mod. Phys. E 6, 693 (1997)

29. S. Peris and E. de Rafael, Phys. Lett. B 309, 389 (1993)

30. K. Dannbom, L. Ya. Glozman, C. Helminen, and D.O. Riska, Nucl. Phys. A616, 555 (1997)

31. I. S. Gerstein and B. W. Lee, Phys. Rev. 152, 1418 (1966)

32. X. Song, J.S. McCarthy, and H.J. Weber, Phys. Rev. D 55, 2624 (1997)

33. J. Ellis and M. Karliner, Phys. Lett. B, 341, 397 (1995)

34. Particle Data Group, R.M. Barnett et al., Review of Particle Physics, Phys. Rev. D 54, 1 (1996)

35. M. Bourquin et al., Z. Phys. C 12, 307 (1982); 21, 1 (1983); 21, 17 (1983); 21, 27 (1983)

36. J.-M. Gaillard and G. Sauvage, Ann. Rev. Nucl. Part. Sci. 34, 351 (1984) 


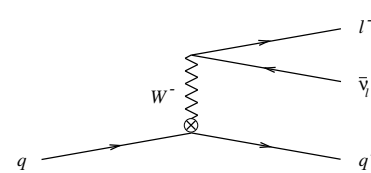

(a)

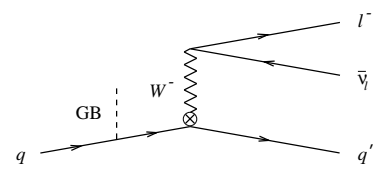

(c)

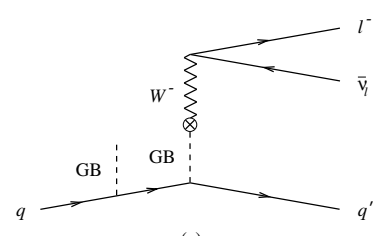

(e)

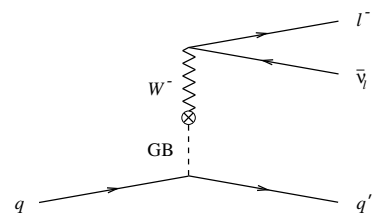

(b)
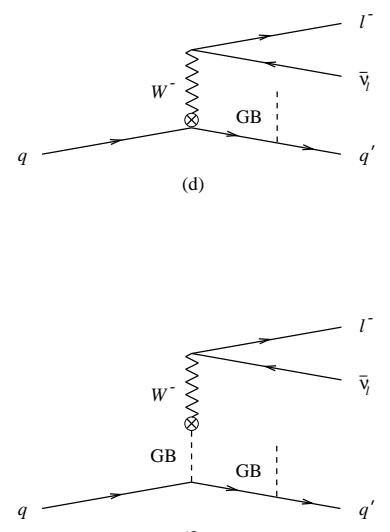

(f)
Table 2. Weak axial-vector form factors, $G_{A}^{B B^{\prime}}$. The values in the NQM column are the $\mathrm{SU}(6)$ values for the weak axialvector form factors and the values in the $\chi \mathrm{QM}$ column are obtained from the quark spin polarizations given in Table 1 . $g_{1}^{\mathrm{QM}} \Sigma^{\Sigma^{ \pm}} \Lambda$ are given instead of $G_{A}^{\Sigma^{ \pm} \Lambda}$, since $f_{1}^{\mathrm{QM}^{\Sigma^{ \pm} \Lambda}}=0$

\begin{tabular}{lrr}
\hline Quantity & $\mathrm{NQM}$ & $\chi \mathrm{QM}$ \\
\hline$G_{A}^{n p}$ & $\frac{5}{3}$ & 1.12 \\
$G_{A}^{\Sigma^{-} \Sigma^{0}}$ & $\frac{2}{3}$ & 0.45 \\
$g_{1}^{\mathrm{QM}} \Sigma^{ \pm} \Lambda$ & $\sqrt{\frac{2}{3}}$ & 0.55 \\
$G_{A}^{\Xi^{-} \Xi^{0}}$ & $-\frac{1}{3}$ & -0.22 \\
\hline$G_{A}^{\Sigma^{-} n}$ & $-\frac{1}{3}$ & -0.22 \\
$G_{A}^{\Xi^{-}} \Sigma^{0}$ & $\frac{5}{3}$ & 1.12 \\
$G_{A}^{\Xi^{-}}$ & $\frac{1}{3}$ & 0.22 \\
$G_{A}^{\Lambda p}$ & 1 & 0.67 \\
$G_{A}^{\Xi^{0}} \Sigma^{+}$ & $\frac{5}{3}$ & 1.12 \\
\hline
\end{tabular}

Fig. 1. Diagrams contributing to the transition $q \rightarrow q^{\prime}+l^{-}+\bar{\nu}_{l}$. $\mathbf{a}, \mathbf{b}$ are zeroth order diagrams with respect to $f_{\Phi}, \mathbf{c}-\mathbf{f}$ are first order diagrams with respect to $f_{\Phi}$

Table 1. Quark spin polarizations. $\Delta \Sigma$ is the total quark spin polarization, i.e. $\Delta \Sigma=\Delta u+\Delta d+\Delta s$. The experimental values have been obtained from [33]. The data for the NQM and the $\chi \mathrm{QM}$ can been obtained from [13, 15

\begin{tabular}{lrrr}
\hline Quantity & Experimental value & NQM & $\chi \mathrm{QM}$ \\
\hline$\Delta u$ & $0.83 \pm 0.03^{\mathrm{a}}$ & $\frac{4}{3}$ & 0.79 \\
$\Delta d$ & $-0.43 \pm 0.03^{\mathrm{a}}$ & $-\frac{1}{3}$ & -0.32 \\
$\Delta s$ & $-0.10 \pm 0.03^{\mathrm{a}}$ & 0 & -0.10 \\
$\Delta \Sigma$ & $0.31 \pm 0.07^{\mathrm{a}}$ & 1 & 0.37 \\
\hline
\end{tabular}

${ }^{\mathrm{a}}$ Obtained with $g_{A}^{n p} \approx 1.26$ and $\Delta u+\Delta d-2 \Delta s \approx 0.60$ 
Table 3. The weak form factors $f_{i}$ and $g_{i}$, where $i=1,2,3$ in the $\chi \mathrm{QM}$.

\begin{tabular}{lcccccc}
\hline & & & & & & \\
Decay & $f_{1}$ & $f_{2}$ & $f_{3}$ & $g_{1}$ & $g_{2}$ & $g_{3}$ \\
\hline$n \rightarrow p$ & 1.00 & 3.52 & $0^{\mathrm{a}}$ & 1.12 & $0^{\mathrm{a}}$ & -210 \\
$\Sigma^{-} \rightarrow \Sigma^{0}$ & 1.41 & 1.84 & $0^{\mathrm{a}}$ & 0.63 & $0^{\mathrm{a}}$ & -190 \\
$\Sigma^{-} \rightarrow \Lambda$ & 0 & 2.73 & 0.10 & 0.55 & -0.25 & -240 \\
$\Sigma^{+} \rightarrow \Lambda$ & 0 & 2.72 & 0.09 & 0.55 & -0.22 & -210 \\
$\Xi^{-} \rightarrow \Xi^{0}$ & 1.00 & -2.27 & $0^{\mathrm{a}}$ & -0.22 & $0^{\mathrm{a}}$ & 83 \\
\hline$\Sigma^{-} \rightarrow n$ & -1.00 & 1.82 & 0.84 & 0.22 & -0.03 & -7.2 \\
$\Xi^{-} \rightarrow \Sigma^{0}$ & 0.71 & 2.72 & -0.44 & 0.79 & 0.28 & -29 \\
$\Xi^{-} \rightarrow \Lambda$ & 1.22 & -0.07 & -0.93 & 0.27 & 0.01 & -10 \\
$\Lambda \rightarrow p$ & -1.22 & -1.68 & 0.62 & -0.82 & -0.10 & 21 \\
$\Xi^{0} \rightarrow \Sigma^{+}$ & 1.00 & 3.83 & -0.62 & 1.12 & 0.42 & -41 \\
\hline
\end{tabular}

${ }^{\mathrm{a}}$ The mass difference for baryons in the same isospin multiplet has been neglected

Table 4. The ratios $\rho_{f}^{B B^{\prime}} \equiv \frac{f_{2}^{B B^{\prime}}}{f_{1}^{B B^{\prime}}}$. The experimental values have been obtained from [35] (see also [36]). The CVC results use as input the experimental values of the anomalous magnetic moments $\mu_{p}^{a} \equiv \mu_{p}-1 \approx 1.793 \mu_{N}$ and $\mu_{n}^{a} \equiv \mu_{n} \approx-1.913 \mu_{N}$, where $\mu_{N}$ is the nuclear magneton. $f_{2}^{\Sigma^{ \pm} \Lambda}$ are given instead of $\rho_{f}^{\Sigma^{ \pm}} \Lambda$, since $f_{1}^{\Sigma^{ \pm} \Lambda}=0$

\begin{tabular}{|c|c|c|c|c|c|c|c|c|}
\hline Quantity & $\begin{array}{l}\text { Experimental } \\
\text { value }\end{array}$ & CVC & RQM 㝳 & MIT [5] & LAPP 8] & QCD 9 & $\chi \mathrm{QSM}_{\mathrm{br}}$ & $\chi \mathrm{QM}$ \\
\hline$\rho_{f}^{n p}$ & $3.71 \pm 0.00$ (input) & 3.71 & 3.62 & 3.63 & 2.95 & 3.71 (input) & 3.16 & 3.52 \\
\hline$\rho_{f}^{\Sigma^{-} \Sigma^{0}}$ & - & 0.84 & - & 1.35 & - & - & 0.86 & 1.30 \\
\hline$f_{2}^{\Sigma^{-} \Lambda}$ & $3.52 \pm 3.52$ & 2.34 & 2.67 & 2.79 & 2.33 & 2.48 & 2.57 & 2.73 \\
\hline$f_{2}^{\Sigma^{+} \Lambda}$ & - & 2.34 & - & - & - & - & - & 2.72 \\
\hline$\rho_{f}^{\Xi^{-} \Xi^{0}}$ & - & -2.03 & - & - & - & - & - & -2.27 \\
\hline$\rho_{f}^{\Sigma^{-} n}$ & $\begin{array}{c}-1.78 \pm 0.61 \\
-1.71 \pm 0.27\end{array}$ & -2.03 & -1.95 & -2.04 & -1.72 & -2.39 & -2.11 & -1.82 \\
\hline$\rho_{f}^{\Xi^{-} \Sigma^{0}}$ & - & 3.71 & - & 4.92 & 3.33 & 5.12 & 3.92 & 3.84 \\
\hline$\rho_{f}^{\Xi^{-} \Lambda}$ & $-0.44 \pm 0.46$ & -0.12 & - & 0.14 & -0.17 & 0.16 & -0.33 & -0.06 \\
\hline$\rho_{f}^{\Lambda p}$ & $2.43 \pm 1.49$ & 1.79 & 1.98 & 1.90 & 1.14 & 2.44 & 1.36 & 1.38 \\
\hline$\rho_{f}^{\Xi^{0} \Sigma^{+}}$ & - & 3.71 & - & - & - & - & - & 3.83 \\
\hline
\end{tabular}

Table 5. The ratios $\rho_{g}^{B B^{\prime}} \equiv \frac{g_{2}^{B B^{\prime}}}{g_{1}^{B B^{\prime}}}$. The experimental value has been obtained from [3]

\begin{tabular}{|c|c|c|c|c|c|c|c|}
\hline Quantity & $\begin{array}{l}\text { Experimental } \\
\text { value }\end{array}$ & RQM 叫 & MIT 5 & LAPP 8 & QCD 9 & $\chi \mathrm{QSM}_{\mathrm{br}} 10$ & $\chi \mathrm{QM}$ \\
\hline$\overline{\rho_{g}^{n p}}$ & - & -0.45 & 0 & - & 0 (input) & 0 & $0^{a}$ \\
\hline$\rho_{g}^{\Sigma^{-} \Sigma^{0}}$ & - & - & 0 & - & - & 0 & $0^{\mathrm{a}}$ \\
\hline$\rho_{g}^{\Sigma^{-} \Lambda}$ & - & -0.46 & -0.03 & -0.12 & -0.27 & -0.06 & -0.46 \\
\hline$\rho_{g}^{\Sigma^{+} \Lambda}$ & - & - & -0.03 & - & - & - & -0.41 \\
\hline$\rho_{g}^{\Xi^{-}} \Xi^{0}$ & - & - & - & - & - & - & $0^{\mathrm{a}}$ \\
\hline$\rho_{g}^{\Sigma^{-} n}$ & $-5.0 \pm 3.9$ & -0.72 & 0.27 & -0.55 & -0.37 & 0.12 & -0.14 \\
\hline$\rho_{g}^{\Xi^{-} \Sigma^{0}}$ & - & - & 0.42 & -0.25 & -0.03 & 0.18 & 0.36 \\
\hline$\rho_{g}^{\Xi^{-} \Lambda}$ & - & - & 0.37 & -0.47 & -0.24 & 0.05 & 0.05 \\
\hline$\rho_{g}^{\Lambda p}$ & - & -0.66 & 0.29 & -0.32 & -0.15 & 0.12 & 0.12 \\
\hline$\rho_{g}^{\Xi^{0} \Sigma^{+}}$ & - & - & - & - & - & - & 0.37 \\
\hline
\end{tabular}

${ }^{\mathrm{a}}$ The mass difference for baryons in the same isospin multiplet has been neglected 
Table 6. The weak form factors $g_{A}^{B B^{\prime}}$ and $g_{P T}^{B B^{\prime}}$. The experimental values for $g_{A}^{B B^{\prime}}$ have been obtained from [34, except for the $g_{1}^{\Sigma^{-} \Lambda}$ and $g_{A}^{\Xi^{-} \Sigma^{0}}$ values, which are CERN WA2 [35, 36] results from branching ratio measurements. The experimental values all assume that the weak form factor $g_{2}=0$. The experimental value for $g_{P T}^{\Sigma^{-}}{ }^{n}$ has been obtained from 3

\begin{tabular}{|c|c|c|c|c|}
\hline Decay & $\begin{array}{c}g_{A}^{B B^{\prime}} \\
\text { Experimental value }\end{array}$ & $\chi \mathrm{QM}$ & $\begin{array}{c}\qquad g_{P T}^{B B^{\prime}} \\
\text { Experimental value }\end{array}$ & $\chi \mathrm{QM}$ \\
\hline$n \rightarrow p$ & $1.2601 \pm 0.0025$ (average) & 1.12 & - & $0^{\mathrm{a}}$ \\
\hline$\Sigma^{-} \rightarrow \Sigma^{0}$ & - & 0.45 & - & $0^{\mathrm{a}}$ \\
\hline$\Sigma^{-} \rightarrow \Lambda^{\mathrm{b}}$ & $\left(0.589 \pm 0.016^{\mathrm{c}}(\mathrm{CERN}\right.$ WA2 $\left.)\right)$ & 0.55 & - & -0.25 \\
\hline$\Sigma^{+} \rightarrow \Lambda^{\mathrm{b}}$ & - & 0.55 & - & 0.22 \\
\hline$\Xi^{-} \rightarrow \Xi^{0}$ & - & -0.22 & - & $0^{\mathrm{a}}$ \\
\hline$\Sigma^{-} \rightarrow n$ & $\begin{array}{c}-0.20 \pm 0.08^{\mathrm{d}} \\
-0.340 \pm 0.017 \text { (average) }\end{array}$ & -0.22 & $1.00 \pm 0.66$ & 0.03 \\
\hline$\Xi^{-} \rightarrow \Sigma^{0}$ & $\left(1.25 \pm 0.15^{\mathrm{c}}(\mathrm{CERN}\right.$ WA 2$\left.)\right)$ & 1.12 & - & 0.40 \\
\hline$\Xi^{-} \rightarrow \Lambda$ & $0.25 \pm 0.05$ (average) & 0.22 & - & 0.01 \\
\hline$\Lambda \rightarrow p$ & $0.718 \pm 0.015$ (average) & 0.67 & - & 0.08 \\
\hline$\Xi^{0} \rightarrow \Sigma^{+}$ & - & 1.12 & - & 0.41 \\
\hline
\end{tabular}

${ }^{\mathrm{a}}$ The mass difference for baryons in the same isospin multiplet has been neglected

${ }^{\mathrm{b}} g_{1}^{\Sigma^{ \pm} \Lambda}$ and $g_{2}^{\Sigma^{ \pm} \Lambda}$ are given instead of $g_{A}^{\Sigma^{ \pm} \Lambda}$ and $g_{P T}^{\Sigma^{ \pm} \Lambda}$, respectively, since $f_{1}^{\Sigma^{ \pm} \Lambda}=0$

${ }^{\mathrm{c}}$ Not listed in the Review of Particle Physics [34]

${ }^{\mathrm{d}}$ Evaluated using $g_{P T}^{\Sigma^{-}}{ }^{n}=1.00 \pm 0.66$ 